\title{
$\eta$ Carinae: The 1998 brightening and the smearing-out effect
}

\author{
A. M. van Genderen ${ }^{1}$ and C. Sterken ${ }^{2, \star}$ \\ 1 Leiden Observatory, Postbus 9513, 2300RA Leiden, The Netherlands \\ e-mail: genderen@strw.leidenuniv.nl \\ 2 Vrije Universiteit Brussel (VUB), Pleinlaan 2, 1050 Brussels, Belgium
}

Received 15 March 2004 / Accepted 11 June 2004

\begin{abstract}
A comparison of broad-band photometry of the Homunculus (ground-based data) and of broad-band and narrowband photometry of the central region (HST-based) from 1997 to 2003 reveals that, apart from the brightening in 1998, the Homunculus as well as the central star varied in a very similar way. This excludes smearing-out effects as an explanation for the difference between the brightening of the central region and that of the Homunculus during 1998. A plausible reason for the homologous light behaviour is enhanced extinction decrease in the line-of-sight to the central region during a global brightening occurring after the 1998.0 spectroscopic event. From 1998 to 2004 there is indeed a brightness excess of $\sim 0.3$ between the Homunculus and the extrapolated secular brightening. We also illustrate that ground-based aperture photometry of the Homunculus is a reliable diagnostic tool for studying the variability of the central star.
\end{abstract}

Key words. technique: photometric - stars: individual: $\eta$ Carinae - variables - supergiants

\section{Introduction}

It is not possible to separate the light of $\eta$ Carinae's central star (CS) from that of the surrounding nebula (major axis 17") using ground-based photometry. The Homunculus, radiating by nearly pure reflection, consists of two hollow lobes. The CS is imbedded in a circumstellar dust shell and is viewed through the wall of the SE lobe. As the CS is variable, the Homunculus is expected to vary as well. van Genderen et al. (1995) used the metaphor of the Chinese lantern to illustrate that the flickering of the nebula should be in concert with changes in the central region (CR: diameter about $0{ }^{\prime} 1 \sim 220 \mathrm{AU}$ encompassing the CS), since light-time effects are expected to be small - though some reduction of the amplitudes is to be expected (van Genderen et al. 1999). Serious doubt on this picture arose by the brightening of the CR that was seen shortly after the 1998.0 event (the supposed periastron passage of a binary). The CR doubled its flux while the Homunculus brightened by 30\% only (Davidson et al. 1999; Sterken et al. 1999a, 2001a).

The ability of the Homunculus to partly mask the optical variations of the $\mathrm{CR}$ was a matter of some concern to Smith et al. (2000). These authors also found that many small gas and dust condensations varied their apparent brightness often in a way very different from the variations of the illuminating source. Since then, it was tacitly assumed that ground-based aperture photometry of the Homunculus is an unreliable diagnostic tool for unraveling the variability of the CS.

\footnotetext{
^ Research Director, Belgian Fund for Scientific Research (FWO).
}

Below we give support to the hypothesis that the difference in the 1998 brightening between CR and Homunculus was due to an excess extinction in the line-of-sight which had nothing to do with smearing-out effects. These smearing-out effects are negligible anyway.

\section{Analysis of available photometric data}

Figure 1 shows, schematically, broad-band photometry of the $\mathrm{CR}$ and the Homunculus from various sources. The $V$ scale is approximately on the $U B V$ system as obtained by various authors: as such the graph should not be used for reading off $V$ magnitudes since not all these data are strictly on the same photometric system (for the intricacies in standardising $\eta$ Car photometry, see Sterken et al. 1999a, 2001).

Figure 1 also shows the magnitudes (+) of the CR obtained in 2003 in the F550M stellar continuum band (Martin et al. 2004) shifted by $-2 \mathrm{~m} 08$ so that the middle data points fit the ascending branch of the continuous curve. The time series of Sterken et al. (1999a) are given as averages, but suggest a superimposed oscillation (amplitude $\sim 0.05$ ) like so many other S Dor variables exhibit. Such oscillations, unavoidably, add to the intrinsic scatter in Fig. 1.

Figure 2 shows the $U$ and $V$ narrow-band light curves between JD2452600 and JD2452910 in more detail (+: the $F 330 W$ and $F 550 M$ magnitudes were shifted by $-2^{\mathrm{m}} 04$ and $+5^{\mathrm{m}} \cdot 39$, respectively). The good match of the $V$ light curves of the CR and of the Homunculus between 2002.8 and 2003.6 is surprising. Their undulatory similarity excludes any smearingout effect as an explanation for what happened in 1998-1999: between 1999 and 2003 all data points are confined to a 


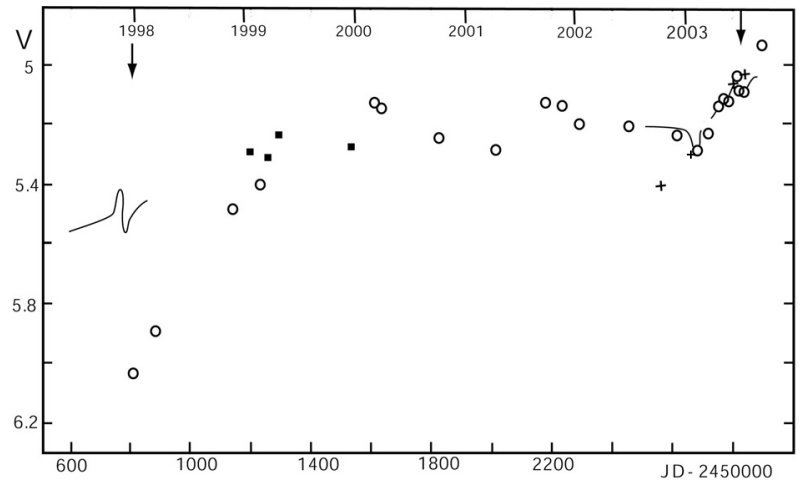

Fig. 1. The combination of schematic light curves from various groups of observers between 1997 and 2004. The $V$ scale is close to the $U B V$ system. The arrows at the top indicate the computed events using the ephemeris by Corcoran (2003). For the Homunculus: short full curve on the left is from van Genderen et al. (1999); black squares: mean of time series of Sterken et al. (1999b); short curve on the right: van Genderen et al. (2003b) and Lajús et al. (2003). For the CR: ○ and + represent daily averages from Martin et al. (2004) shifted along the magnitude axis by -2 m 08 for a good match between their 2002.8-2003.6 curve and the short light curve to the right.

relatively narrow band which supports the view that the low brightness level of the CR in 1998-1999 was not normal.

Davidson et al. (1999) discussed the possibility that in 1998 the line-of-sight extinction to the CR decreased more than it did in other regions. The area up to $1^{\prime \prime}(\sim 2250 \mathrm{AU})$ around the CR also showed a steeper brightening than the Homunculus did between the two data points of June 1997 and June 1999 (in the narrow-band filters $F 336 \mathrm{~W}$ and $F 631 \mathrm{~W}$ ), which is hard to explain, see Smith et al. (2000). Because of the absence of smearing-out effects, an extinction decrease is a possibility: there must have been additional dust extinction along the line-of-sight relatively close to the CS, which disappeared in 1998-1999 together with a global, but smaller decrease of circumstellar extinction. In Fig. 1 the decrease of the excess extinction over the first four data points of the CR shows a linear trend with a gradient of $-0.0015 \mathrm{~d}^{-1}$ or $-0.55 \mathrm{y}^{-1}$ and an extrapolation of this line suggests that somewhere between 1999.3 and 2000.0 there was no excess extinction left.

The very first narrow-band magnitude of the CR (JD $2452561,+$ sign in Fig. 1) appears about 0.2 fainter than the remaining data. One could speculate that, just like in 1998, an excess extinction is present in the line-of-sight, an excess that has disappeared two months later on JD 2452624 (o, the $V$ broad-band magnitude of the $\mathrm{CR}$ ) and that remained absent thereafter. However, then one would expect a magnitude difference increasing towards shorter wavelengths between first and second narrow-band data point at JD 2452682 (the second one - the brighter of the two - is expected to be normal as supported by the good match seen in Figs. 1 and 2). This is not the case, the differences are $0 \mathrm{~m} 12$ in F550M, 0.02 in $F 330 \mathrm{~W}, 0 \mathrm{~m} 08$ in F250W, $0 \mathrm{~m} 12$ in $F 220 \mathrm{~W}$. Thus, the first F330W-magnitude shows a brightness excess. It is perhaps no coincidence that its date (JD 2452 561) is only 9 days ahead from the predicted maximum in the Balmer continuum as obtained from solution 1 for the "200-d oscillation" (van Genderen et al. 1999, 2001)

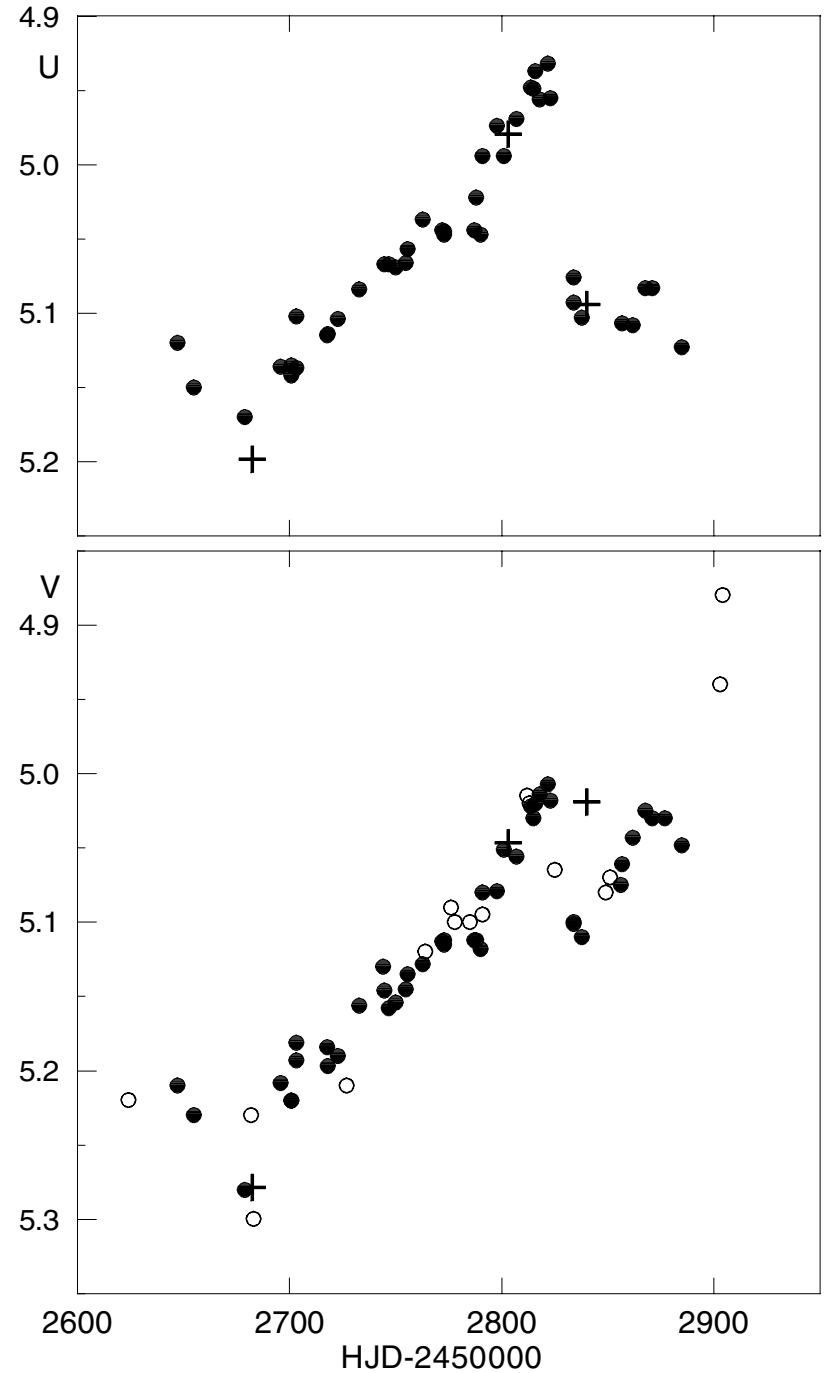

Fig. 2. The detailed light curves between 2002.8 and 2003.6. For the Homunculus: • from van Genderen et al. (2003b). For the CR: $\circ$ and + are the same as in Fig. 1. The $U$ and $V$ HST narrow-band magnitudes were shifted by -2 m. 04 and +5 . 39 , respectively.

and $30 \mathrm{~d}$ behind for solution 2 . Some associated activity in the Fe II emission lines, which abundantly occur in the two other UV filters, is probably the reason, and explains why the differences are not larger than in the visual band. Thus, our conjecture is that we are dealing with a new case of an extinction excess in the line-of-sight to the CR.

In contrast to the UV data ( $F 330 \mathrm{~W}$ band), the last visual narrow-band data point (JD 2452840, F550M band) in Fig. 2 did not show the eclipse-like dip. Obviously, this dip is very wavelength-dependent, as the F550M band is much narrower than the $V$ band. The progressively increasing depth of the dip towards shorter wavelengths suggests that a light source is obscured by gas (like, for example, an extended atmosphere of a companion), or by the interface of colliding winds.

Right after the 1998.0 PP the colour of the CR became bluer (Davidson et al. 1999; Smith et al. 2000), as well as the $b-y$ of the Homunculus (Sterken et al. 1999, 2001). Davidson et al. (1999) and Smith et al. (2000) attribute this nearly grey extinction to large dust particles. Caution is called 


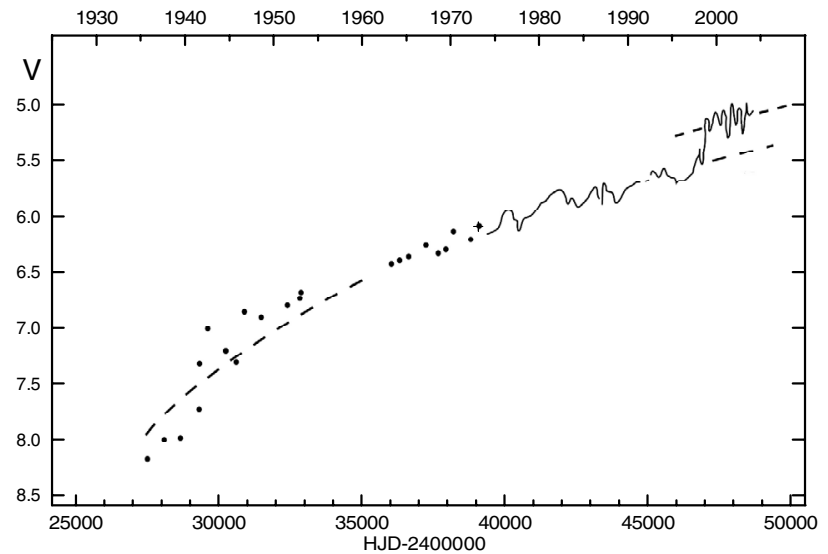

Fig. 3. The secular rise in the visual since 1935: dashed curve (van Genderen et al. 1994). The $V$ scale is on the $U B V$ system. The wavy continuous curve represents the monitored observations since 1974.

for, first of all because small particles are likely present in the central area (Robinson et al. 1987; Smith et al. 1998) and small particles predominantly occur in the entire Homunculus (King et al. 2002): as such, the blueing should have been much stronger. Secondly, because this so-called grey extinction can also be partially caused by the ongoing $\mathrm{S}$ Dor phase, which started mid 1996 with a pronounced brightening and associated reddening (as it should) in most Geneva colour indices (van Genderen et al. 1999) and in $b-y$ (by $\sim 0$. 1 , Sterken et al. 1999a) until the 1998.0 PP. From this moment on the $b-y$ colour index of the Homunculus became suddenly bluer while the brightness rise continued at least at the same rate, i.e. between JD 2450880 and JD $2451200 \mathrm{~V}$ became brighter by 0.18 and $b-y$ became bluer by 0.03 only. The underlying $\mathrm{S}$ Dor phase, if still underway to a light maximum (it is unlikely that the SD-phase reversed precisely at 1998.0) must have annihilated part of the blueing.

We like to comment upon the last CR observation after the 2003.5 PP (2003.7, Figs. 1 and 2), which suggests that there was another brightness peak, like after the 1986.9 PP (Fig. 3 in van Genderen et al. 2003a). A short series of $V$ measurements of the Homunculus made on 31 December 2003 indicates that the brightness has declined by $0 \mathrm{~m} 1$, and the peak was apparently fading ( $V=5.03$, Allen 2004).

Figure 3 shows the secular brightening since 1935 (van Genderen et al. 1994). The dashed curve represents the observed trend, which we assume is mainly due to the decrease of the extinction by the expanding Homunculus. Note that the dusty lobes are supposed to be largely responsible for the obscuration of their own scattered light (van Genderen et al. 1995). The quasi-continuous wavy curve schematically represents the brightness fluctuations 1974-2003 of the Homunculus.

Obviously, the 1998.0 PP marks the start of the unusual permanent (at least up to 2004) brightening of the Homunculus by $\sim 0$. 3 on the average. Besides, the 1999-2003 curve suggests the same rate of secular brightening, but at a higher level (short dashed line). We believe that during the 1998.0 PP something happened on or near the CS resulting in a global brightening. The visual brightness difference between $\mathrm{CR}$ and Homunculus was 2.8 in 1991 (based on $U B V$ data obtained close to the HST data of the CR, van Genderen et al. 2003a). The secular brightening would have decreased this difference to $2^{\mathrm{m}} 6$ early 1999 , while it was 2 . 4 as derived from the data of Martin et al. (2004) and Sterken et al. (1999a). This is consistent with the expected 2.3 difference. If the 0.3 brightening was the consequence of a possible change in bolometric magnitude of the CS, that change would also be about 0.3 since the effective temperature remains about constant and the Homunculus is expected to mimic the CS. However, a luminosity increase must have thinned the dust shells in the vicinity of the CS. Therefore, the global brightening could be due to either a combination of a luminosity increase and a thinning of dust (as also advocated by Smith et al. 2000), or by a thinning of the dust alone (see Sect. 3).

\section{Conclusions}

By a comparison of the photometric observation of the CR by the HST and of the Homunculus between 1997 and 2004, we conclude that the Homunculus mimics the CR very well. Smearing-out effects, whether by a different response of dust and gas condensations to the brightness variations of the CR and by other masking effects, or by light-time effects, are negligible.

We conclude that the much stronger brightening of the CR during 1998 compared to that of the Homunculus can be ascribed to an additional extinction decrease in the line-of-sight. The fact that the blueing in 1998 was much less than expected can partly be explained by the ongoing $S$ Dor phase (since 1996) on its way to a maximum in light and red colour, cancelling part of the blueing by extinction decrease. When interpreting the light and colour variation of the CR and the Homunculus, the intrinsic variations of the CS should not be neglected.

The new case of the brightness deficit of the CR at the end of 2003, if real, questions the rarity of the phenomenon. If indeed dust is condensing in the stellar envelope at such a very high rate as the abundance analysis of Viotti \& Rossi (1999) suggests, then one should observe in the long run a gradual increase of the extinction again, until a new equilibrium is reached.

Acknowledgements. This work has been supported by "IUAP P5/36" Interuniversity Attraction Poles Programme of the Belgian Federal Science Policy, and the Belgian Fund for Scientific Research (FWO).

\section{References}

Allen, W. H. 2004, private communication

Corcoran, M. F. 2003, Website modified 16 September 2003 http://lheawww.gsfc.nasa.gov/users/corcoran/eta_car.htlm

Corcoran, M. F., Ishibashi, K., Swank, J. H., \& Petre, R. 2001, ApJ, 547,1034

Davidson, K., Humphreys, R. M., Ishibashi, K., et al. 1999, AJ, 118, 1777

van Genderen, A. M., Sterken, C., de Groot, M., et al. 1995, A\&A, 304, 415 
van Genderen, A. M., Sterken, C., de Groot, M., \& Burki, G. 1999, Smith, N., Morse, J., Davidson, K., \& Humphreys, R. M. 2000, AJ, A\&A, 343, 847 120,920

van Genderen, A. M., de Groot, M., \& Sterken, C. 2001, ASP Conf. Ser., 233, 55

Sterken, C. 2003, ASP Conf. Ser., 292, 437

van Genderen, A. M., Sterken, C., \& Allen, W. A. 2003a, A\&A, 405, 1057

Sterken, C., de Groot, M., \& van Genderen, A. 1996, A\&AS, 116, 9

Sterken, C., Freyhammer, L. M., Arentoft, T., \& van Genderen, A. M. 1999a, A\&A, 346, L33

van Genderen, A. M., Sterken, C., Allen, W. H., \& Liller, W. 2003b, A\&A, 412, L25

King, N. L., Nota, A., Walsh, J. R., et al. 2002, ApJ, 581, 285

Lajús, E. F., Gamen, R., Schwartz, M., et al. 2003, IBVS 5477

Martin, J. C., Koppelman, M. D., et al. 2004, AJ, in press

Robinson, G., Mitchell, R. M., Aitken, D. K., et al. 1987, MNRAS, 227,535

Sterken, C., van Genderen, A. M., de Groot, M., \& Burki, G. 1999b, ASP Conf. Ser., 179, 200

Sterken, C., Freyhammer, L. M., Arentoft, T., et al. 2001, ASP Conf. Ser., 233, 71

Viotti, R., \& Rossi, C. 1999, in Eta Carinae at the millennium, ed. J. A. Morse, R. M. Humphreys, \& A. Damineli, ASP Conf. Ser., 179, 211

Smith, N., Gehrz, R. D., \& Krautter, J. 1998, AJ, 116, 1332 